\title{
Overexpression of autocrine motility factor in metastatic tumor cells: possible association with augmented expression of KIF3A and
} GDI- $\beta$

\author{
Takashi Yanagawa $^{1}$, Hideomi Watanabe ${ }^{1}$, Toshiyuki Takeuchi ${ }^{2}$, Shuhei Fujimoto ${ }^{3}$, \\ Hideyuki Kurihara ${ }^{2,4}$ and Kenji Takagishi ${ }^{1}$ \\ ${ }^{1}$ Department of Orthopaedic Surgery; ${ }^{2}$ Department of Molecular Medicine; ${ }^{3}$ Department of Microbiology and \\ ${ }^{4}$ Department of Neurosurgery Gunma University Faculty of Medicine, Institute for Molecular and Cellular \\ Regulation, Gunma University, Showa, Maebashi, Gunma 371-8511, Japan
}

\begin{abstract}
Autocrine motility factor (AMF), which is identical to phosphohexose isomerase (PHI)/glucose-6-phosphate isomerase (GPI), a ubiquitous enzyme essential for glycolysis, neuroleukin (NLK), a neurotrophic growth factor, and maturation factor (MF) mediating the differentiation of human myeloid cells, enhances the motility and metastatic ability of tumor cells. AMF/PHI activity is elevated in the serum or urine in patients with malignant tumors. Here, we constructed an amf/phi/nlk/mf gene using adenovirus vector and transfected into two tumor cell lines. Overexpression of AMF/PHI/NLK/MF enhanced AMF secretion into the culture media in both tumor cell lines. However, upregulation of motility and metastatic ability was found only in metastatic fibrosarcoma cells expressing an AMF receptor, gp78, and was not found in gp78-undetectable osteosarcoma cells. Thus, not only serum AMF activity but also gp78-expression in tumor cells may be required for metastasis-related motility induction. With the use of microarray analyses, we detected two augmented genes, rho GDP dissociation inhibitor beta and kinesin motor 3A, as well as AMF itself. The RNA message and protein expression of these two molecules was confirmed to be upregulated, suggesting a possible association with AMF-induced signaling for cell motility and metastasis.

Laboratory Investigation (2004) 84, 513-522, advance online publication, 16 February 2004; doi:10.1038/labinvest.3700057
\end{abstract}

Keywords: AMF/PHI/GPI/NLK; KIF3A; GDI- $\beta$; motility; metastasis

One of the major features of malignancy is the invasive potential of the tumor cells. Liotta ${ }^{1}$ proposed a three-step hypothesis for the mechanism of tumor cell invasion. The first is tumor cell attachment via cell surface receptors to the matrix, the second is local degradation of the matrix, and the third is tumor cell locomotion into the region of the matrix. A group of secreted cytokines inducing cell motile activity in vitro may initiate cell locomotion in vitro. ${ }^{2}$ Among them, autocrine motility factor (AMF) stimulates cell motility in various types of tumor cells in an autocrine manner. ${ }^{3,4}$ We purified AMF from conditioned medium of murine metastatic fibrosarcoma, and demonstrated that AMF is

Correspondence: Dr H Watanabe, MD, PhD (DMSc), Department of Orthopaedic Surgery, Gunma University Faculty of Medicine, 3-39-22 Showa, Maebashi, Gunma 371-8511, Japan.

E-mail: hidewa@showa.gunma-u.ac.jp

Received 14 April 2003; revised 18 June 2003; accepted 8

September 2003; published online 16 February 2004 identical to phosphohexose isomerase (PHI), also designated as glucose-6-phosphate isomerase (GPI), which is a ubiquitous enzyme catalyzing the conversion of glucose-6-phosphate to fructose-6-phosphate. ${ }^{5} \mathrm{AMF} / \mathrm{PHI}$ has also been found to be identical to neuroleukin (NLK), ${ }^{6,7}$ a neurotrophic growth factor that supports the survival of spinal and sensory neurons, ${ }^{8}$ and maturation factor (MF) mediating the differentiation of human myeloid leukemic HL-60 cells to terminal monocytic cells, ${ }^{9}$ suggesting that this cytokine is multifunctional, probably dependent on the target cells. AMF/PHI activities have shown to be elevated in the serum or urine of patients with disseminated malignant tumors such as gastrointestinal, kidney, breast, colorectal, and lung carcinomas, ${ }^{10,11}$, thereby being useful as a tumor-dissemination marker. An immunohistochemical study has demonstrated that the prognosis of AMF/PHI-positive patients with pulmonary adenocarcinoma is poorer than that of negative patients. ${ }^{12}$ Furthermore, AMF/PHI was 
found in rheumatoid joints, ${ }^{13,14}$ and was implicated as a synovial self-pathogenic antigen responsible for the onset of rheumatoid arthritis. ${ }^{15,16}$

$\mathrm{AMF}$ binds to a unique $78 \mathrm{kDa}$ glycoprotein receptor, and initiates gp78 phosphorylation., ${ }^{4,17}$ Anti-gp78 monoclonal antibody (3F3A) stimulates motility of metastatic tumor cell, ${ }^{18}$ and reveals the localization of gp78 on the leading lamella as well as the trailing edge of motile cells. ${ }^{19}$ The expression of gp78 is a predicting factor for prognosis in patients with various malignant neoplasms including esophageal, colorectal, bladder, gastric, and lung carcinoma. ${ }^{20-25}$

The molecular pathway and regulatory mechanisms by which AMF manifests its effect on cells via its receptor have been elucidated in part. The signaling pathway involves receptor phosphorylation, ${ }^{4}$ and the inhibitory effect of pertussis toxin (PT) on the AMF-mediated cell motility suggests a regulatory role of G-binding protein. ${ }^{26}$ It is also involved in the activation of phospholipases and the inositol cycle ${ }^{27}$ with two downstream components, 12-lipoxygenases and protein kinase C. ${ }^{28}$ Analysis of the protein kinase cascade(s) in the AMF-stimulated cell motility using specific kinase inhibitors reveals the involvement of protein kinase $\mathrm{C}$ and tyrosine kinase, but not kinase $\mathrm{A}$, reiterating that the signaling is independent of the adenylate cyclase pathway. ${ }^{29}$ Recently, AMF stimulation has been shown to result in stress-fiber formation, which is associated with activation of two family members of the Rholike GTPases, RhoA and Rac1, but not Cdc42 activation. This association of the specific small GTPase Rho activation is accompanied by AMFinduced redistribution of the small GTPase from a uniform cellular distribution to the lamellipodia and filopodia at the leading edges of the cells' periphery. ${ }^{30}$ Interestingly, transfection of AMF/PHI-tagged hemagglutinin into Cos7 cells reveals its intracellular localization within actin-rich pseudopodial domains. ${ }^{31}$ Torimura et $a l^{32}$ have demonstrated that AMF/PHI enhances Rho activity and phosphorylation of MEK1 and MEK2 in human hepatoma cell lines. Rho is one of the most important molecules in the AMF/PHI signal transduction.

In this study, to elucidate the metastatic mechanisms induced by AMF, we constructed an amf/phi transfection system using adenovirus, and then investigated its secretion, effects on cell motility, and metastasis. Furthermore, the expression of the molecules related to downstream reaction of AMF/ PHI stimulation was analyzed with microarray analysis.

\section{Materials and methods}

\section{Cells and Culture Conditions}

To investigate the function of $\mathrm{AMF} / \mathrm{PHI}$ as an autocrine cytokine, we used two cell lines able to grow in a protein-free condition. One was a murine fibrosarcoma cell line, Gunma clone-4 protein-free (Gc-4 PF), ${ }^{5}$ in which the motility and metastatic capacity show high responses to AMF by increased expression of the receptor. ${ }^{18}$ Another was Dunn osteosarcoma protein free (DPF), a mouse osteosarcoma cell line. ${ }^{33}$ Gc-4 PF cells were cultured in RPMI 1640 medium without serum. DPF cells were cultured in RPMI 1640 medium including trace elements $\left(5 \mu \mathrm{M} \mathrm{FeCl}, 5 \mu \mathrm{M} \quad \mathrm{ZnSO}_{4}\right.$, and $10 \mu \mathrm{M}$ sodium potassium tartrate) without serum. Both cells were incubated at $37^{\circ} \mathrm{C}$ in a humidified atmosphere of $5 \% \mathrm{CO}_{2}$ in air.

\section{Construction of Recombinant Adenovirus}

The cDNA of AMF/PHI was cloned by RT-PCR using mRNA extracted from BALB/cAnNCrj mouse liver as a template and the following primers: $5^{\prime}$-CTTCCGAGCACGTCCTGC- $3^{\prime}$ and $5^{\prime}$-CTAGC TGGGGTGTGAAATACAG- ${ }^{\prime}$. The PCR product was inserted into TA cloning vector pcR2.1 (Invitrogen Corp., Carlsbad, CA, USA), cut with NotI and HindIII, and inserted into pcDNA3 vector (Invitrogen Corp., Carlsbad, CA, USA) containing CMV promoter lesion. The whole sequence of the insert was analyzed and contained no mutation. An expression unit was excised with NruI and SmaI and inserted into the $S w a$ I site of the cassette cosmid pAdex1cw. The DNA sample was packaged using Gigapack XL (Stratagene, La Jolla, CA, USA). After plating the transduced Escherichia coli, we could obtain a clone containing the desired insert. Recombinant adenovirus was constructed by homologous recombination in human embryonic kidney cell line 293 cells using the DNA samples and the EcoT22I-digested DNA-terminal protein complex (DNA-TPC). Viruses were propagated in the 293 cells and purified by two rounds of CsCl density centrifugation. We constructed two kinds of vectors, one of which contained lacZ gene (AdlacZ) and the other amf/phi gene (Adamf/phi). The cells were infected by viral solutions to cell monolayers and incubated at $37^{\circ} \mathrm{C}$ for $1 \mathrm{~h}$ with brief agitation every $20 \mathrm{~min}$. Culture medium was added, and the infected cells were returned to the $37^{\circ} \mathrm{C}$ incubator.

\section{$\beta$-Galactosidase Transduction Assay}

To assess the efficiency of the adenovirus-mediated gene transfer, $\beta$-galactosidase activity of Gc- $4 \mathrm{PF}$ and DPF cells infected by adenovirus at 10 multiplicity of infection (MOI) and $100 \mathrm{MOI}$ was examined 2 and 7 days after infection. At each point, cells were washed two times with PBS, fixed with $0.25 \%$ glutaraldehyde, washed four times with PBS, and stained in a $1 \mathrm{mg} / \mathrm{ml}$ 5-bromo-4-chloro-3-indolyl$\beta$ - $D$-galactopyranoside (X-gal) solution consisting of $0.2 \mathrm{mM} \mathrm{MgCl}_{2}, 5 \mathrm{mM} \mathrm{K} \mathrm{K}_{3}\left[\mathrm{Fe}(\mathrm{CN})_{6}\right]$ and $5 \mathrm{mM}$ $\mathrm{K}_{4}\left[\mathrm{Fe}(\mathrm{CN})_{6}\right]$ in PBS. $\beta$-Galactosidase-positive cells in each well were counted microscopically. 


\section{Immunoblot Analysis}

Immunoblot analysis was performed to detect AMF/ PHI and the receptor, gp78 in Gc-4 PF1 and DPF cells, as described previously. ${ }^{5}$ Briefly, cells were cultured on 100-mm dishes, infected by adenovirus at 10 and $100 \mathrm{MOI}$ when they were confluent, and $200 \mu \mathrm{l}$ lysis buffer (PBS, 0.5\% NP-40, 2 mM PMSF, $1 \mathrm{mM}$ EDTA) was added 2 days after infection. The samples were incubated on ice for $30 \mathrm{~min}$, centrifuged at $12000 \mathrm{~g}$ for $5 \mathrm{~min}$, and the supernatants were collected. At the same time, conditioned media were collected, dialyzed, lyophilized, and diluted with PBS. Samples were analyzed by SDS-PAGE in polyacrylamide slab gels at reducing condition for AMF receptor, gp78, and nonreducing condition for $\mathrm{AMF}$, and electrotransferred to transfer membranes. The membranes were incubated with anti-AMF polyclonal antibody 1:400 in PBS containing 15\% low-fat milk. Lactoperoxidase-labeled anti-rabbit antibody (Vector, Burlingame, CA, USA) was used for detection. ECL Western blotting system, an enhanced chemiluminescence detection system (Amersham Pharmacia Biotech, Uppsala, Sweden), was then used to detect the antibody-specific proteins bound to the transfer membranes, according to the manufacturer's instructions.

To examine the upregulation of protein expression in response to amf/phi gene overexpression, transfected Gc-4 PF cells were lysed in NP-40 lysis buffer, 1\% (v/v) NP-40/50 mM Tris, pH 8.0/150 mM $\mathrm{NaCl}$, containing leupeptin $(10 \mu \mathrm{g} / \mathrm{ml})$, aprotinin $(10 \mu \mathrm{g} / \mathrm{ml})$, and phenylmethanesulfonyl fluoride ( $1 \mathrm{mM}$ ), incubated on ice for $30 \mathrm{~min}$, centrifuged at $12000 \mathrm{~g}$ for $5 \mathrm{~min}$, and then the supernatants were collected. Subsequent steps were the same as mentioned above. Anti-GDI- $\beta$ goat polyclonal antibody (Santa Cruz Biotechnology, Inc., CA, USA) and antiKIF3A mouse antibody (BD Biosciences, San Jose, CA, USA) were used for detection of each molecule.

\section{Phagokinetic Tracks}

Gc-4 PF1 and DPF cells were infected by adenovirus at 10 and $100 \mathrm{MOI} 2$ days before assay. Uniform carpets of gold particles were prepared on coverslips coated with bovine serum albumin, as described previously. ${ }^{34}$ Colloidal gold-coated coverslips were placed in 35-mm tissue culture dishes, and 3000 cells in suspension culture were added to the plates. After $24 \mathrm{~h}$, the phagokinetic tracks were visualized using dark-field illumination in a Nikon inverted microscope. The area cleared of gold particles by at least 50 cells was measured using NIH image 1.62, and the standard error reflecting a 95\% confidence level was calculated.

\section{Experimental Pulmonary Metastasis}

To examine the ability of experimental metastasis formation, 20000 cells infected by adenovirus at 10 and $100 \mathrm{MOI}$ were injected intravenously at the tail vein into $\mathrm{C} 3 \mathrm{H} / \mathrm{He}$ mice. Mice were killed 3 weeks later, and the lungs were examined for lungcolonizing nodules.

\section{Oligonucleotide Microarray Analysis}

Gc-4 PF1 cells were separated into three conditions; infected by Adamf/phi or adenovirus alone at 100 MOI where infection rate was almost $100 \%$, or not infected. The isolated total RNA of each cell 2 days after transfection was used for synthesis of cDNA, which was labeled with Cy3 and hybridized with Atlas Glass Array Mouse 1.0 (Clontech Laboratories, Inc., Palo Alto, CA, USA) containing probe sets for 1081 mouse genes. The signal intensities from hybridized cDNA were quantified, and the ArrayGauge (Fuji film, Tokyo, Japan) analysis software was used to identify differentially expressed genes. The genes whose expression was up- and downregulated more than two-fold in response to adenovirus-mediated overexpression of AMF/PHI were selected and analyzed.

\section{RT-PCR Analysis}

RT-PCR was performed to confirm upregulation of RNA messages of the genes suggested to be upregulated in the microarray analysis. Total RNA was extracted from cells using Isogen (Wako, Osaka, Japan) 2 days after transfection. A measure of $3 \mu \mathrm{g}$ of total RNA treated with DNase was used as a template for cDNA synthesis. The products of $\mathrm{RT}$ reactions were used for PCR. Genes examined here involved rho GDP dissociation inhibitor beta (GDI- $\beta$ ) and kinesin motor 3A (KIF3A). The housekeeping gene glyceraldehyde-3-phosphate dehydrogenase (GAPDH) was used as a control. The number of cycles, 25 times for each gene, was selected to allow linear amplification of the cDNA under study. The primers sequences and their respective PCR fragment lengths were as follows: KIF3A, 5'-ACGCT GACGACATGGATAGAATCATG-3', $5^{\prime}$-CAGTTTGG AGTTCCGATACGGCAC-3' (335bp); GDI- $\beta$, 5'-CAGA CCCAACAGTTCCCAATGTGAC-3', 5'-CCATGAATG TGGCTTTATCCACTCTC-3' (249bp); and GAPDH 5'CAAGATGGTGAAGGTCGGTGT-3', 5' ${ }^{\prime}$-GGGTTTCTT ACTCCTTGGAGG-3' (1013 bp). The abundance of the PCR products of interest was expressed in pixel intensities and divided for the abundance of the GAPDH signal amplified in parallel from the same cDNA sample.

\section{Immunofluorescence Analysis Investing KIF3A Distribution}

Gc-4 PF1 cells plated for $24 \mathrm{~h}$ on glass coverslides were transfected with Adamf/phi gene or adenovirus alone at $100 \mathrm{MOI}$. After $48 \mathrm{~h}$, cells were fixed with chilled methanol, incubated with anti-KIF3A antibody, and then the distribution was visualized 
with FITC-conjugated rabbit anti-mouse IgG (CEDERLANE, Hornby, Ont., Canada). The coverslips bearing the stained cells were mounted in $90 \%$ glycerol in PBS for viewing and photomicrography.

\section{Statistical Analysis}

Phagokinetic and metastasis assays were analyzed with Bonferroni/Dunn's method as analysis of variance (ANOVA) and post hoc test.

\section{Results}

\section{Adenovirus-mediated LacZ Expression in Gc-4 PF1 and DPF Cells}

The proportions of $\beta$-galactosidase activity-positive Gc-4 PF1 and DPF cells infected AdlacZ at $100 \mathrm{MOI}$ were both $100 \%$ at 2 days after infection (Figure 1a and e) and $95.8 \pm 2.7$ and $100 \%$ at 7 days after infection, respectively (Figure $1 \mathrm{~b}$ and f). On the other hand, the proportions of positive cells at $10 \mathrm{MOI}$ were $17.0 \pm 6.1$ and $54.5 \pm 9.8 \%$ at 2 days after infection (Figure 1c and g) and $14.4 \pm 2.6$ and $53.0 \pm 10.8 \%$ at 7 days after infection, respectively (Figure $1 \mathrm{~d}$ and $\mathrm{h}$ ). There were no positive cells when Adamf/phi, adenovirus alone, or nothing was transfected (data not shown).

\section{Adenovirus-mediated Intracellular and Extracellular Expression of AMF/PHI and gp78 in Gc-4 PF and DPF Cells}

The expression of intra- and extracellular AMF/PHI was detected in Gc-4 PF1 cells under all conditions (Figure 2a and b). Both intracellular and extracellular expressions were augmented by the infection with Adamf/phi at $100 \mathrm{MOI}$. Interestingly, dose dependency was evident in extracellular expression (Figure 2b). Although the intracellular AMF/PHI expression pattern in DPF cells was similar to that in Gc-4 PF1 cells (Figure 3a), the extracellular molecules were detected only in cells infected by Adamf/ phi at $100 \mathrm{MOI}$ (Figure $3 \mathrm{~b}$ ).

The expression of gp78 was found in Gc-4 PF cells as described previously, ${ }^{18}$ and was augmented by transfection with Adamf/phi in a dose-dependent manner (Figure 4a). In contrast, no expression of gp78 was observed in DPF cells even in the cells transfected by amf/phi gene at $100 \mathrm{MOI}$ (Figure 4b), suggesting absence of the receptor for AMF in DPF cells.

\section{Effects of Transfection of amf/phi Gene on Motility of Gc-4 PF1 and DPF Cells}

As shown in Figure 5a, transfection of the amf/phi gene stimulates motility of Gc-4 PF1 cells in a dosedependent manner. The motility of Gc-4 PF1 cells infected by Adamf/phi at 100 MOI was significantly
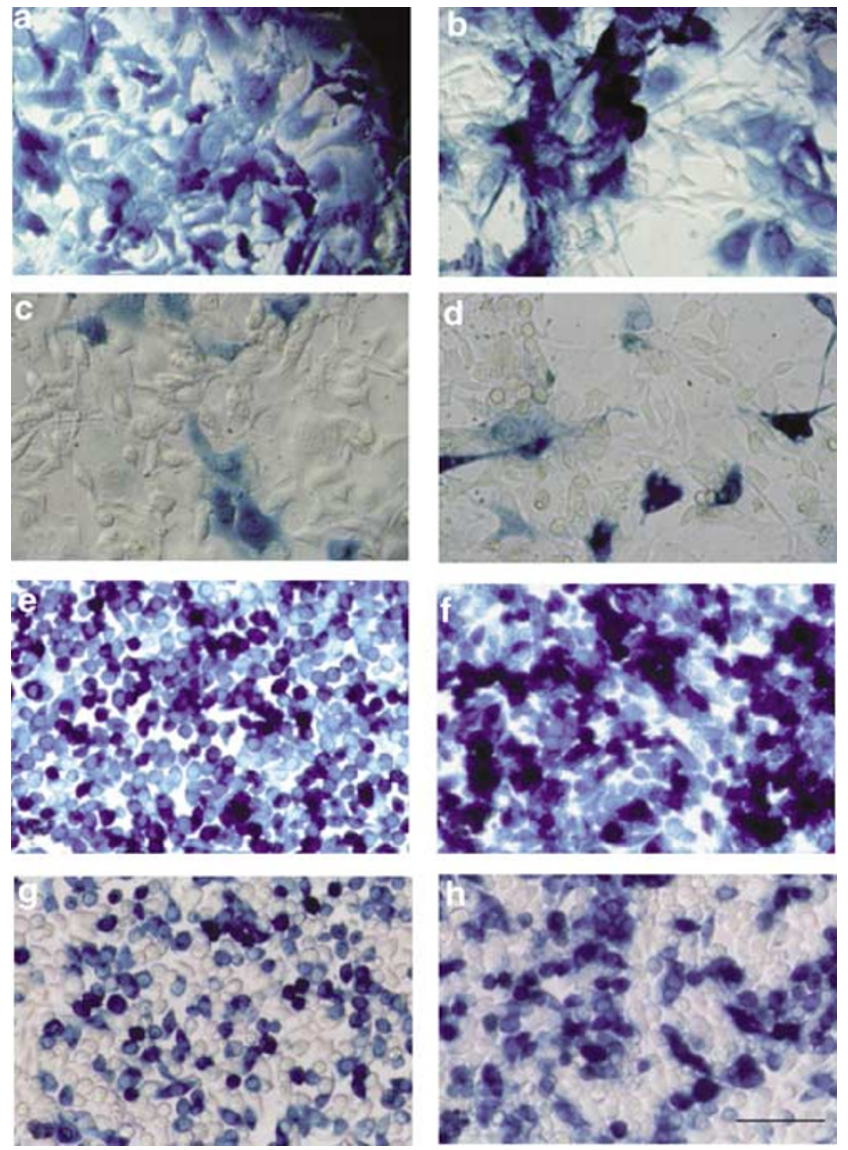

Figure 1 Adenovirus-mediated LacZ expression. Gc-4 PF1 ((a)(d)) and DPF cells ((e)-(h)) were infected with AdlacZ at 100 MOI $(\mathbf{a}, \mathbf{b}, \mathbf{e}, \mathbf{f})$ and at $10 \mathrm{MOI}(\mathbf{c}, \mathbf{d}, \mathbf{g}, \mathbf{h}) . \quad \beta$-Galactosidase activity was examined $2(\mathbf{a}, \mathbf{c}, \mathbf{e}, \mathbf{g})$ or 7 days $(\mathbf{b}, \mathbf{d}, \mathbf{f}, \mathbf{h})$ after infection. Positive cells were depicted as blue cells. Bar indicates $20 \mu \mathrm{m}$.

a

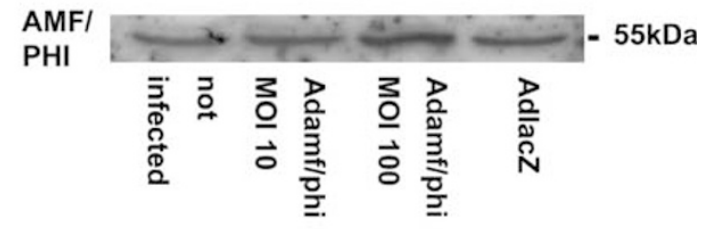

b

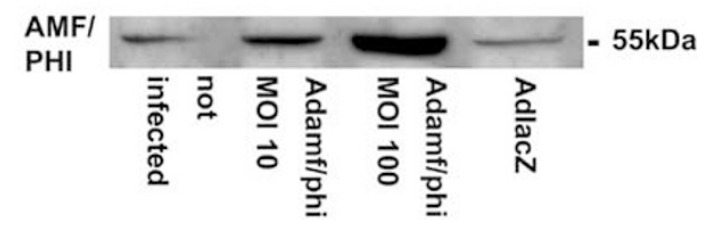

Figure 2 Effect of adenovirus-mediated amf/phi gene transfection on the expression of intracellular (a) and extracellular (b) AMF/ PHI in Gc-4 PF1 cells.

higher than those not infected or infected by AdlacZ (ANOVA; $F(3,125)=9.128, P=0.0003,<0.0001$, respectively), and cells infected by Adamf/phi at 
a

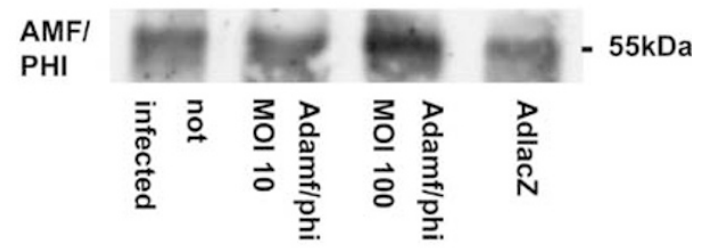

b

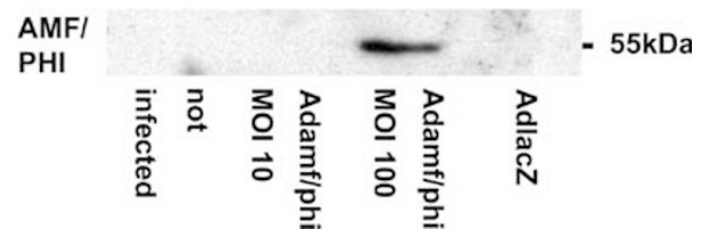

Figure 3 Effect of adenovirus-mediated amf/phi gene transfection on the expression of intracellular (a) and extracellular (b) AMF/ PHI in DPF cells.

a

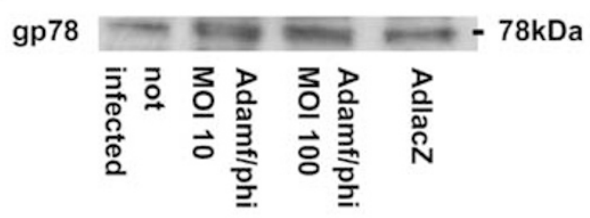

b

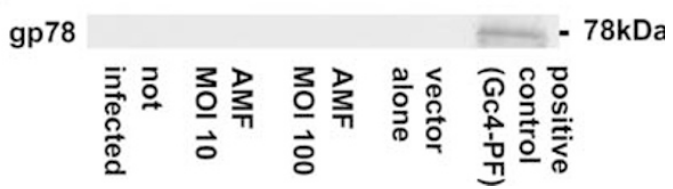

Figure 4 Effect of adenovirus-mediated transfection of amf/phi gene on gp78 expression in Gc-4 PF1 (a) and DPF (b) cells.

10 MOI showed significantly higher motility than cells infected by AdlacZ $(P=0.0024)$. In contrast, there was no change in the phagokinetic activity of DPF cells after the cells were transfected by amf/phi gene using adenovirus vector even at $100 \mathrm{MOI}$ (Figure 5b).

\section{Metastatic Responses of Gc-4 PF1 and DPF Cells to Transfection of amf/phi Gene}

As shown in Figure 6, transfection of amf/phi gene resulted in an increase in the lung metastatic nodules of Gc-4 PF cells in a dose-dependent manner, while there was a negligible increase in the colonization of the AdlacZ-transfected cells. The metastatic ability of the cells infected by Adamf/ phi at $100 \mathrm{MOI}$ was significantly higher than that of untransfected and AdlacZ-transfected cells (ANOVA; $F(3,28)=6.833, P=0.001$ and 0.0008 , respectively). a

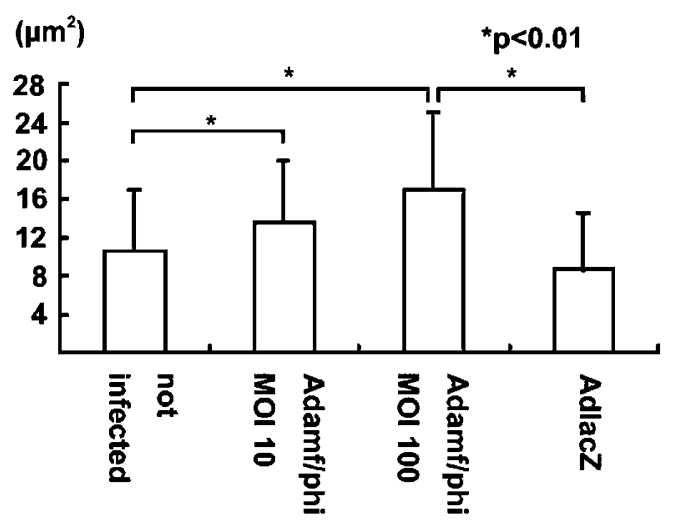

b

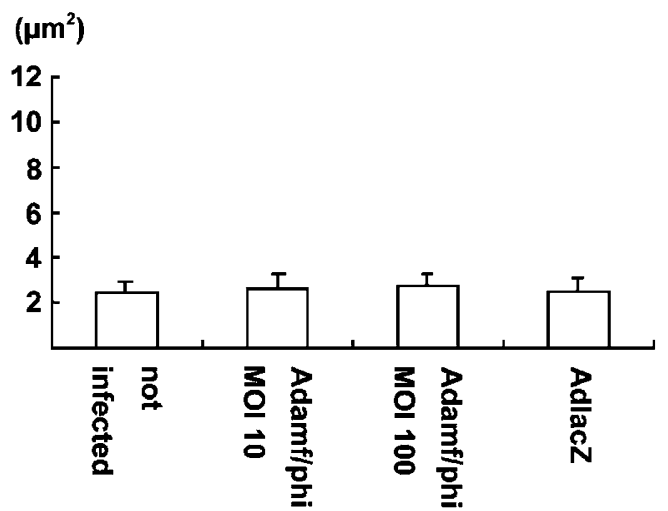

Figure 5 Effect of transfection of amf/phi gene on cell motility. Gc-4 PF1 (a) and DPF cells (b). transfected without or with Adamf/phi, or AdlacZ were plated on colloidal gold-coated coverslips. The area cleared of gold particles by at least 50 cells was photographed, and measured using NIH image 1.62.

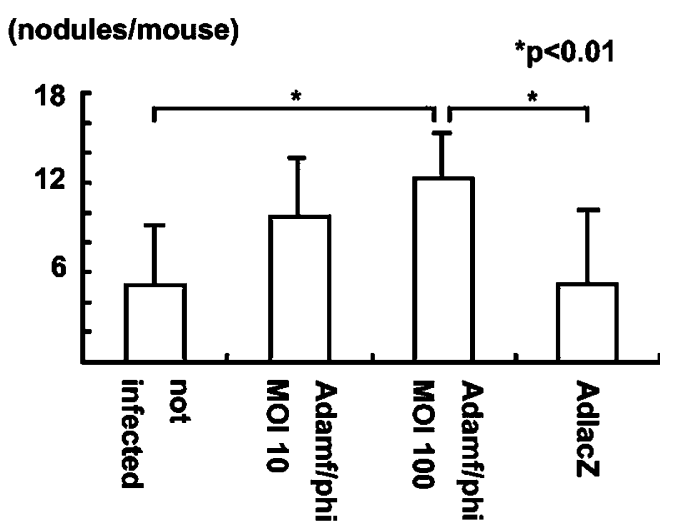

Figure 6 Metastatic ability of Gc-4 PF1 cells induced by transfection of amf/phi gene. Mice were given i.v. injections of $2 \times 10^{4}$ Gc- 4 PF1 cells transfected without or with Adamf/phi, or AdlacZ. The average number of lung-colonizing nodules is shown.

In contrast to Gc-4 PF cells, no metastatic pulmonary nodules were found after intravenous injection of DPF even after transfection of Adamf/ phi at 100 MOI. 


\section{Identification of Genes Induced by amf/phi Gene Transfection by Microarray Analysis}

Out of 1081 mouse-related genes, 14 and seven genes whose expression in the cells infected by Adamf/phi gene were increased and decreased more than two-fold compared with untransfected cells were, respectively, identified (Table 1). From them, three genes whose expression was more than twofold compared with that of cells infected with adenovirus alone were selected. These were considered to be unaffected by adenovirus itself, and no suppressed genes were found in this selection. The identified genes were rho GDP dissociation inhibitor beta (GDI- $\beta$ /GDID4/LyGDI; GenBank accession No. L07918), microtubule plus end-directed kinesin motor 3A (KIF3A; GenBank accession No. D12645), and phosphohexoseisomerase (PHI/AMF/NLK/MF; GenBank accession No. M14220). The enhanced rates of transfection of $\mathrm{amf} / \mathrm{phi}$ gene against adenovirus alone were $2.15,2.24$, and 4.68 , respectively.

\section{Augmented Expression of GDI- $\beta$ and KIF3A Transfected by amf/phi Gene Using RT-PCR and Immunoblot Analyses}

RT-PCR analysis showed augumented expression of KIF3A after amf/phi gene transfection to be almost two-fold the mRNA level as compared with adenovirus transfection as well as nontransfection (Figure $7 a)$. Immunoblot analysis also showed that a $27-\mathrm{kDa}$ band expression was augmented in amf/phi genetransfected cells (Figure 7b), suggesting that overexpression of amf/phi gene induces protein production as well as RNA message of KIF3A. On the other hand, since augmented RNA message of GDI- $\beta$ induced by amf/phi gene transfection was less than twice that of the control, we performed statistical analysis from three independent experiments. Densitometric analysis revealed that the intensity ratios of GDI- $\beta$ expression against GAPDH expression in amf/phi gene-transfected, vector alone-transfected, and nontransfected cells were 1.122, 0.683, and 0.529 , respectively, and that transfection of amf/phi resulted in significantly higher expression than the others (ANOVA; $F(2,9)=9.850, \quad P=0.0114$, $<0.0021$, respectively) (Figure 7c). Immunoblot analysis also showed again that an $80-\mathrm{kDa}$ band was augmented in amf/phi gene-transfected cells (Figure 7d). These results confirmed those obtained from microarray analyses.

\section{KIF3A Distribution in Gc-4 PF Cells}

KIF3A is a microtubule-related protein, ${ }^{35}$ and AMF receptor, gp78, is a family of microtubule-associated tubular organelles. ${ }^{36}$ These findings prompted us to investigate the effect of amf/phi transfection on the KIF3A distribution. In the perinuclear area of untransfected cells, small puncta or tubulovesicular structures were abundant, as shown in neuron

Table 1 Up- and downregulated genes by infection of adenovirus including amf/phi gene in more than two-fold as compared with those in untransfected cells

\begin{tabular}{|c|c|c|}
\hline Gene name & Rate & $\begin{array}{l}\text { GenBank } \\
\text { accession } \\
\text { no. }\end{array}$ \\
\hline \multicolumn{3}{|l|}{ Upregulated genes } \\
\hline Homeobox protein 2.4 (HOX2.4) & 3.11 & $\mathrm{X} 13721$ \\
\hline Interferon regulatory factor 2 (IRF2) & 2.19 & J03168 \\
\hline myc proto-oncogene & 2.76 & X01023 \\
\hline $\begin{array}{l}\text { Glucose-6-phosphate isomerase (GPI); phosphoglucose isomerase (PGI); phosphohexoseisomerase (PHI); } \\
\text { neuroleukin (NLK) }\end{array}$ & 4.02 & M14220 \\
\hline Endothelin 3 & 2.16 & U32330 \\
\hline Neuromodulin; growth-associated protein 43 (GAP43); protein F1; calmodulin-binding protein p57 & 2.43 & J02809 \\
\hline Mothers against dpp homolog 7 (SMAD7; MADH7); MÅDH8 & 2.71 & AF015260 \\
\hline Rho GDP dissociation inhibitor beta (GDI-beta; ARHGDIB); GDP dissociation inhibitor D4 (GDID4) & 2.69 & L07918 \\
\hline Matrix metalloproteinase 14 (MMP14); membrane-type Matrix matalloproteinase 1 (MTMMP1) & 3.23 & X83536 \\
\hline Tissue inhibitor of metalloproteinase 3 (TIMP3 ); SUN & 2.61 & L19622 \\
\hline Microtubule plus end-directed kinesin motor 3A (KIF3A) & 4.96 & D12645 \\
\hline Alpha internexin neuronal intermediate filament protein (alpha-INX; INA) & 2.19 & L27220 \\
\hline Filensin; beaded filament structural protein in lens 1 (BFSP1) & 2.24 & Y13602 \\
\hline Unconventional myosin VI & 3.45 & U49739 \\
\hline \multicolumn{3}{|l|}{ Downregulated genes } \\
\hline Groucho gene-related protein (GRG); amino enhancer of split protein (AES) & 0.43 & L12140 \\
\hline GATA-binding protein 4 (GATA4) & 0.47 & M98339 \\
\hline Ephrin A2 (EFNA2); eph-related receptor tyrosine kinase ligand 6 (EPLG6; LERK6); ELF1; CEK7 ligand (CEK7-L) & 0.39 & U14752 \\
\hline Wingless-related MMTV integration site 3a protein (WNT3A) & 0.35 & X56842 \\
\hline Radical fringe homolog (RFNG) & 0.47 & U94350 \\
\hline crk proto-oncogene & 0.34 & S72408 \\
\hline Transducin beta- 2 subunit & 0.29 & U34960 \\
\hline
\end{tabular}

Increased expression of genes more than two-fold as compared with that in cells transfected with adenovirus alone are depicted in bold. 
a

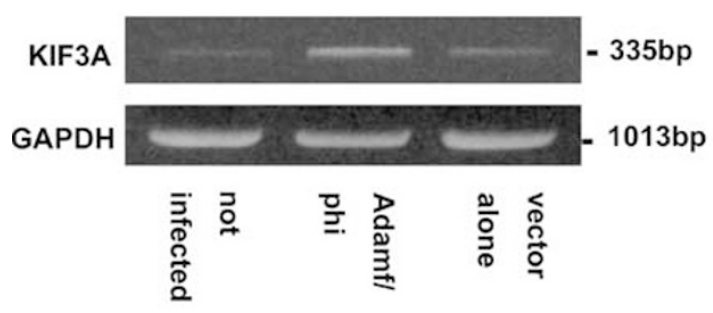

b

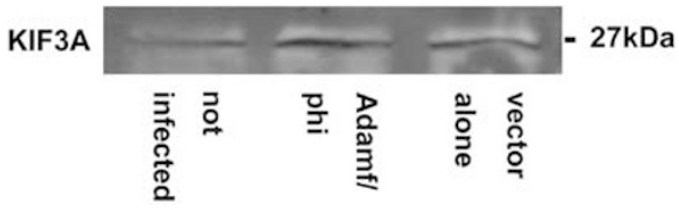

C

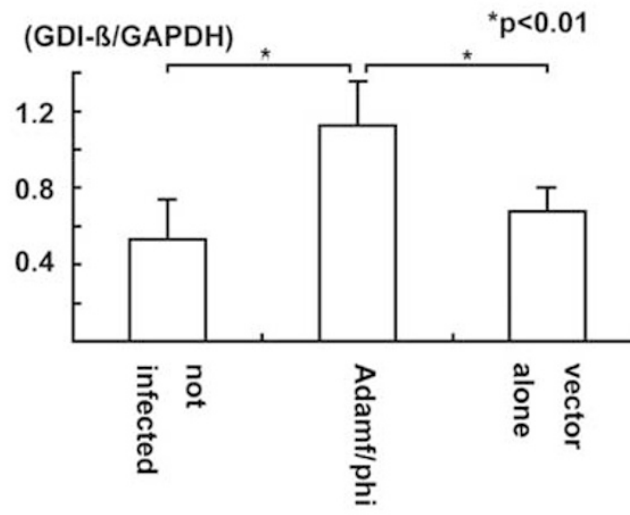

d

GDI-ß

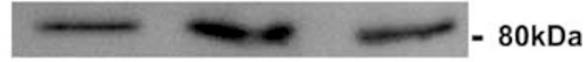

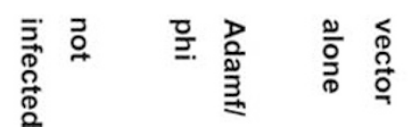

Figure 7 RT-PCR and immunoblot analyses of Gc-4 PF1 cells transfected with $a m f / p h i$ gene. mRNA ((a), (c)) and protein ((b), (d)) expressions of KIF 3A ((a), (b)) and GDI- $\beta$ ((c), (d)) were investigated, as described in experimental procedures.

cells. ${ }^{35}$ On the other hand, in some cells a diffuse distribution was depicted at the ruffled edges of the cells, including lamellipodia (Figure 8), showing a character unique to these metastatic fibrosarcoma cells. More cells with stained lamellipodia seemed to be found in $a m f / p h i$ - than vector-transfected cells.

\section{Discussion}

In the present study, we constructed an amf/phi/ $n l k / m f$ transfection system using adenovirus vector, succeeded in obtaining a high efficiency of gene transfection, and produced a sustained gene expression for at least 1 week. This induction was observed in both cell lines in a dose-dependent manner, but more transduction efficiency seemed to be found in

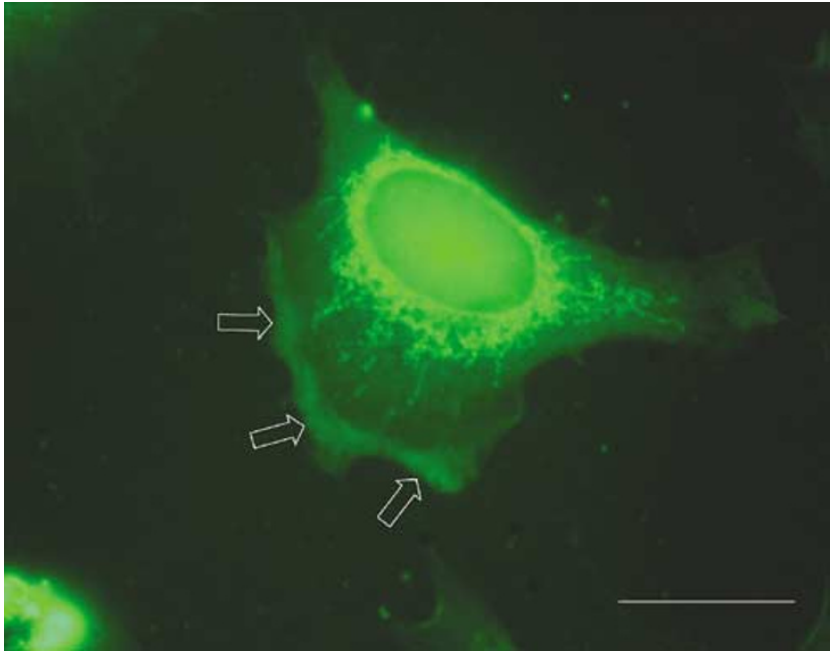

Figure 8 Indirect immunofluorescent labeling of Gc-4 PF cell with the anti-KIF $3 \mathrm{~A}$ mouse anibody. A diffuse distribution was depicted at the ruffled edges of the cells (arrows) in some AMFtransfected cells. Bar indicates $5 \mu \mathrm{m}$.

DPF cells at 10 MOI transfection than in Gc-4 PF cells (half vs one-fifth of total cells), although almost $100 \%$ of the cells were transfected at $100 \mathrm{MOI}$ in both cell lines. Induction of RNA message of delivered amf/phi was clearly demonstrated by microarray analysis. Intracellular protein product was recognized by the anti-AMF pAb in a dosedependent manner. This adenovirus vector-assisted gene delivery system may thus be useful for studying the mechanisms by which AMF/PHI stimulates disseminating ability in metastatic cells.

It is interesting that PHI, which catalyzes the conversion of glucose-6-phosphate to fructose-6phosphate intracellularly, also stimulates extracellularly cell motility in an autocrine manner. The mechanisms of the secretion of AMF/PHI, which lacks a secretary signal peptide, have been elucidated in part. Haga et $a l^{37}$ demonstrated that CK IIphosphorylation contributes to the secretion of AMF via a nonclassical pathway. Niinaka et $a l^{38}$ have described that AMF/PHI is secreted into conditioned medium only in malignant cell lines while both malignant and normal cell lines express AMF/ PHI, and that markedly higher RNA message expression of $\mathrm{AMF} / \mathrm{PHI}$ is seen in malignant than in normal cell lines. In the present study, immunoblot analysis showed that AMF/PHI secretion was augmented by the amf/phi gene transfection in a dose-dependent manner in Gc-4 PF cells. Interestingly, AMF/PHI molecules appeared in conditioned medium following high-amount gene transfection even in DPF cells that did not secrete AMF/PHI before transfection. These results indicated that high production of amf/phi gene RNA message could allow the cells to secrete AMF/PHI even if the cells do not originally secrete AMF/PHI. Such quantitative factors may play an important role in the extracellular secretion of AMF/PHI molecule regardless of 
the cell type, while qualitative factors such as biochemical modification have still not been fully elucidated.

Gc-4 PF1 cells exhibited an increased metastatic ability in response to the transfection of amf/phi gene in a transfection rate-dependent manner. This augmentation of metastatic ability corresponded well to the expression of both AMF/PHI and the receptor, gp78 in this metastatic tumor cell line. On the other hand, in nonmetastatic DPF cells, the metastatic ability was not obtained by gene transfection regardless of evident induction of extracellular AMF/PHI expression. It should be noted that in contrast to Gc-4 PF cells, no expression of gp78 was acquired even after amf/phi gene transfection in DPF cells. Probably, metastatic ability regulated by AMF/PHI may require the receptor, gp78 expression as well as AMF/PHI itself.

It is interesting to elucidate what phenotypes are responsible for augmented metastatic propensity in response to amf/phi gene overexpression. In the present study, augmented cell-motile activity was also found in Gc-4 PF cells in response to the amf/ phi gene transfection in a transfection rate-dependent manner. In contrast, motile activity of DPF cells did not respond to the amf/phi gene transfection. Motility stimulated by AMF/PHI also requires AMFR expression, since gp78 expression was not induced by amf/phi gene transfection in DPF cells. Thus, motility may be most important in the phenotypes regulated by the autocrine AMF/PHI stimulation. Recently, on the other hand, Funasaka et $a 1^{39}$ have demonstrated that AMF/PHI enhances neovascularization and motile activity of human umbilical vein endothelial cells, which would initiate and promote metastasis. AMF/PHI also stimulates MMP-2 secretion and extracellular MMP-2 activation, being related to destruction of the basement membrane followed by metastasis and invasion in hepatoma cells. ${ }^{32}$ These mechanisms occurring in host organs also may contribute to AMF-regulated dissemination of tumor cells.

Rho GTPases play an important role in metastasis of cancer cells. ${ }^{40,41}$ The active form of the Rho family of small GTPases consists of a GTP-bound one, and inactive form GDP-bound. GTPase-activating proteins (GAPs) accelerate intrinsic GTP hydrolytic activity, $^{42}$ guanine nucleotide exchange factors (GEFs) stimulating the replacement of GTP by $\mathrm{GDP}^{43}$ and guanine nucleotide-releasing factors (GRFs), enhancing the GDP dissociation rate. On the other hand, GDP dissociation inhibitors (GDIs) suppress the GDP dissociation rate. ${ }^{44,45}$ In the present study, microarray, RT-PCR, and Western blot analyses revealed that GDI- $\beta$, a member of the Rho GDI superfamily, was upregulated by $a \mathrm{mf} / \mathrm{phi}$ gene transfection. Rho GDI- $\beta$ is preferentially expressed in hematopoietic cells, ${ }^{45}$ and involved in T-cell antigen receptor-induced pathways. ${ }^{46}$ Recently, the structural basis for the regulation of Rho proteins by the molecule has been elucidated. ${ }^{47}$ The role of Rho
GDI- $\beta$ in invasion and metastasis of cancer cells is still controversial at present. Tapper et $a l^{48}$ demonstrated that upregulation of Rho GDI- $\beta$ is associated with progression of ovarian carcinoma with largescale survey in serous ovarian carcinoma by cDNA array analysis, ${ }^{48}$ corresponding well to our present results. In contrast, however, a group of Theodorescu has reported the Rho GDI- $\beta$ as an invasion and metastasis suppressor gene with the findings that the reduced expression is related to metastatic progression of bladder cancer. ${ }^{49-51}$ Interestingly, transfection of Rho GDI- $\beta$ into metastatic cells resulted in suppression of both metastatic ability and in vitro motility, but did not affect in vitro growth, colony formation, or in vivo tumorigenicity. ${ }^{49}$ Upregulation of GDI- $\beta$ in the present study might be induced as a negative signal in the putative feedback mechanisms against excess signals from AMF/PHI. Further detailed experiments will be necessary to elucidate the exact role of the small G-binding protein.

Kinesin is known to be a microtubule-based motility protein that moves to the plus end of microtubules. ${ }^{52}$ Many kinds of kinesin-like motor protein have been identified, all of which have a homologous domain containing a putative ATP and microtubule-binding sites. ${ }^{53}$ KIF3A is one of this superfamily, and forms a complex with KIF3B and kinesin superfamily-associated protein 3 (KAP3). ${ }^{54}$ The role of microtubules in cell adhesion lies in the supporting kinesin-based transport to the adhesion sites, which control the dynamics of adhesive structures. ${ }^{55}$ Adhesion site detachment leading to the retraction of a cell edge in motile cells is associated with microtubule-targeting events. ${ }^{56}$ Recently, kinesin has shown to be required for the focal delivery of possible components to retard substrate adhesions' growth or promote their disassembly. ${ }^{57}$ In the present study, amf/phi-transfected cells showing high motility exhibited not only augmented expression of KIF3A but also a tendency of distribution shift to the cell periphery. KIF3A may play an important role in the focal delivery of a component(s) that modulates the dynamics of adhesive structures at the focal contact, and then stimulate cell motility. Recently, activation of RhoA and Rac1, but not Cdc42 has been shown to be required for AMF/PHI signaling in association with c-Jun kinases. ${ }^{30}$ These molecules corresponded well with components that are possible molecules specifically accumulated at microtubule tips as cofactors, together with cargo delivered by kinesin, in signal transduction events. ${ }^{57}$ Small GTPase rho and the downstream effecter molecules may be possible components to which KIF3A may be related, delivering to the cell periphery in association with microtubules in AMF/PHI-stimulated cells.

In conclusion, $\mathrm{AMF} / \mathrm{PHI}$ overexpression induced AMF/PHI secretion. Augmentation of motility and metastatic ability required not only upregulation of intracellular expression of $\mathrm{AMF} / \mathrm{PHI}$ followed by 
secretion but also sufficient gp78 expression. Overexpression of AMF/PHI may be related to increases in GDI- $\beta$ and KIF3A expression. AMF/PHI is a multipotential molecule. The responses may depend on the cell differentiation. Further investigations will be necessary in order to elucidate the exact role of this molecule in individually differentiated cells.

\section{Acknowledgement}

This work was supported in part by Grants-in-Aid for scientific research (C) 12671395 (HW), and (C) 13671489 (KT) from the Ministry of Education, Science and Culture, Japanese Government.

\section{References}

1 Liotta LA. Tumor invasion and metastases-role of extracellular matrix: Rhoads Memorial Award lecture. Cancer Res 1986;46:1-7.

2 Nabi IR, Watanabe H, Raz A. Autocrine motility factor and its receptor: role in cell locomotion and metastasis. Cancer Metastasis Rev 1992;11:5-20.

3 Liotta LA, Mandler R, Murano, et al. Tumor cell autocrine motility factor. Proc Natl Acad Sci USA 1986;83:3302-3306.

4 Watanabe H, Carmi P, Hogan V, et al. Purification of human tumor cell autocrine motility factor and molecular cloning of its receptor. J Biol Chem 1991;266:13442-13448.

5 Watanabe H, Takehana K, Date M, et al. Tumor cell autocrine motility factor is the neuroleukin/phosphohexose isomerase polypeptide. Cancer Res 1996;56: 2960-2963.

6 Chaput M, Claes V, Portetelle D, et al. The neurotrophic factor neuroleukin is $90 \%$ homolohous with phosphohexose isomerase. Nature 1988;332:454-455.

7 Faik P, Walker JIH, Redmill AAM, et al. Mouse glucose6-phosphate isomerase and neuroleukin have identical $3^{\prime}$ sequences. Nature 1988;332:455-457.

8 Gurney ME, Heinrich SP, Lee MR, et al. Molecular cloning and expression of neuroleukin, a neurotrophic factor for spinal and sensory neurons. Science 1986;234:566-574.

$9 \mathrm{Xu}$ W, Seiter K, Feldman E, et al. The differentiation and maturation mediator for human myeloid leukemia cells shares homology with neuroleukin or phosphoglucose isomerase. Blood 1996;87:4502-4506.

10 Baumann M, Kappel A, Lang T, et al. The diagnostic validity of the serum tumor marker phosphohexose isomerase (PHI) in patients with gastrointestinal, kidney, and breast cancer. Cancer Invest 1990;8: 351-356.

11 Patel PS, Raval GN, Rawal RM, et al. Comparison between serum levels of carcinoembryonic antigen, sialic acid, and phosphohexose isomerase in lung cancer. Neoplasma 1995;42:271-274.

12 Takanami I, Takeuchi K, Naruke M, et al. Autocrine motility factor in pulmonary adenocarcinomas: results of an immunohistochemical study. Tumor Biol 1998; 19:384-389.
13 Takeuchi K, Watanabe H, Takagishi K. Biochemical investigation of cell motile activity in rheumatoid synovial fluid. J Rheumatol 1998;25:9-15.

14 Watanabe H, Takeuchi K, Chigira M. Expression of autocrine motility-like factor in rheumatoid synovial fluid. J Rheumatol 1994;21:37-40.

15 Matsumoto I, Staub A, Benoist C, et al. Arthritis provoked by a linked $\mathrm{T}$ and $\mathrm{B}$ cell recognition of a glycolytic enzyme. Science 1999;286:1732-1735.

16 Schaller M, Burton ER, Ditzel HJ. Autoantibodies to GPI in rheumatoid arthritis: linkage between an animal model and human disease. Nat Immunol 2001; 2:746-753.

17 Shimizu K, Tani M, Watanabe H, et al. The autocrine motility factor receptor gene encodes a novel type of seven transmembrane protein. FEBS Lett 1999;456: 295-300.

18 Watanabe H, Shinozaki T, Raz A, et al. Expression of autocrine motility factor receptor in serum- and protein-independent fibrosarcoma cells: implications for autonomy in tumor-cell motility and metastasis. Int J Cancer 1993;53:689-695.

19 Nabi IR, Watanabe H, Raz A. Identification of B16-F1 melanoma autocrine motility-like factor receptor. Cancer Res 1990;50:409-414.

20 Hirono Y, Fushida S, Yonemura Y, et al. Expression of autocrine motility factor receptor correlates with disease progression in human gastric cancer. $\mathrm{Br} J$ Cancer 1996;74:2003-2007.

21 Maruyama K, Watanabe H, Shiozaki H, et al. Expression of autocrine motility factor in human esophageal squamous cell carcinoma. Int J Cancer 1995;64: 316-321.

22 Nakamori S, Watanabe H, Kameyama $\mathrm{M}$, et al. Expression of autocrine motility factor receptor in colorectal cancer as a predictor for disease recurrence. Cancer 1994;74:1855-1862.

23 Otto T, Birchmeier W, Schmidt U, et al. Inverse relation of E-cadherin and autocrine motility factor receptor expression as a prognostic factor in patients with bladder carcinomas. Cancer Res 1994;54: 3120-3123.

24 Takanami I, Takeuchi K, Watanabe H, et al. Significance of autocrine motility factor receptor gene expression as a prognostic factor in non-small-cell lung cancer. Int J Cancer 2001;95:384-387.

25 Taniguchi K, Yonemura Y, Nojima N, et al. The relation between the growth patterns of gastric carcinoma and the expression of hepatocyte growth factor receptor (c-met), autocrine motility factor receptor, and urokinase-type plasminogen activator receptor. Cancer 1998;82:2112-2122.

26 Stracke ML, Guirguis R, Liotta LA, et al. Pertussis toxin inhibits stimulated motility independently of the adenylate cyclase pathway in human melanoma cells. Biochem Biophys Res Commun 1987;146:339-345.

27 Kohn EC, Liotta LA, Schiffmann E. Autocrine motility factor stimulates a three-fold increase in inositol trisphosphate in human melanoma cells. Biochem Biophys Res Commun 1990;166:757-764.

28 Silletti S, Timar J, Honn KV, et al. Autocrine motility factor induces differential 12-lipoxygenase expression and activity in high- and low-metastatic K-1735 melanoma cell variants. Cancer Res 1994;54: 5752-5756.

29 Kanbe K, Chigira M, Watanabe H. Effects of protein kinase inhibitors on the cell motility stimulated by 
autocrine motility factor. Biochim Biophys Acta 1994;1222:395-399.

30 Tsutsumi S, Gupta SK, Hogan V, et al. Activation of small GTPase Rho is required for autocrine motility factor signaling. Cancer Res 2002;62:4484-4490.

31 Lagana A, Duchaine T, Raz A, et al. Expression of autocrine motility factor/phosphohexose isomerase in Cos7 cells. Biochem Biophys Res Commun 2000;273: 213-218.

32 Torimura T, Ueno T, Kin M, et al. Autocrine motility factor enhances hepatoma cell invasion across the basement membrane through activation of $\beta 1$ Integrin. Hepatology 2001;34:62-71.

33 Yanagawa T, Watanabe H, Shinozaki T, et al. Expression of bone formation-related molecules in a newly established protein-independent osteosarcoma. Int J Oncol 2001;18:1195-1205.

34 Albrecht-Buehler G. The phagokinetic tracks of 3T3 cells. Cell 1997;11:395-404.

35 Kondo S, Sato-Yoshitake R, Noda Y, et al. KIF3A is a new microtubule-based anterograde motor in the nerve axon. J Cell Biol 1994;125:1095-1107.

36 Benlimame N, Simard D, Nabi IR. Autocrine motility factor receptor is a marker for a distinct membranous tubular organelle. J Cell Biol 1995;129:459-471.

37 Haga A, Niinaka Y, Raz A. Phosphohexose isomerase/ autocrine motility factor/neuroleukin/maturation factor is a multifunctional phosphoprotein. Biochim Biophys Acta 2000;1480:235-244.

38 Niinaka Y, Paku S, Haga A, et al. Expression and secretion of neuroleukin/phosphohexose isomerase/ maturation factor as autocrine motility factor by tumor cells. Cancer Res 1998;58:2667-2674.

39 Funasaka T, Haga A, Raz A, et al. Tumor autocrine motility factor is an angiogenic factor that stimulates endothelial cell motility. Biochem Biophys Res Commun 2001;284:1116-1125.

40 Clark EA, Golub TR, Lander ES, et al. Genomic analysis of metastasis reveals an essential role for RhoC. Nature 2000;406:532-535.

41 Jaffe AB, Hall A. Rho GTPases in transformation and metastasis. Adv Cancer Res 2002;84:57-80.

42 Rubinfeld B, Munemitsu S, Clark R, et al. Molecular cloning of a GTPase activating protein specific for the Krev-1 protein p21rap1. Cell 1991;65:1033-1042.

43 Boguski MS, McCormick F. Proteins regulating Ras and its relatives. Nature 1993;366:643-654.

44 Dirac-Svejstrup AB, Sumizawa T, Pfeffer SR. Identification of a GDI displacement factor that releases endosomal Rab GTPases from Rab-GDI. EMBO J 1997; 16:465-472.
45 Scherle P, Behrens T, Staudt LM. Ly-GDI, a GDPdissociation inhibitor of the RhoA GTP-binding protein, is expressed preferentially in lymphocytes. Proc Natl Acad Sci USA 1993;90:7568-7572.

46 Groysman M, Hornstein I, Alcover A, et al. Vav1 and Ly-GDI two regulators of Rho GTPases, function cooperatively as signal transducers in $\mathrm{T}$ cell antigen receptor-induced pathways. J Biol Chem 2002;277: 50121-50130.

47 Scheffzek K, Stephan I, Jensen ON, et al. The RacRhoGDI complex and the structural basis for the regulation of Rho proteins by RhoGDI. Nat Struct Biol 2000;7:122-126.

48 Tapper J, Kettunen E, El-Rifai W, et al. Changes in gene expression during progression of ovarian carcinoma. Cancer Genet Cytogenet 2001;128:1-6.

49 Gildea JJ, Seraj MJ, Oxford G, et al. RhoGDI2 is an invasion and metastasis suppressor gene in human cancer. Cancer Res 2002;62:6418-6423.

50 Harding MA, Arden KC, Gildea G, et al. Functional genomic comparison of lineage-related human bladder cancer cell lines with differing tumorigenic and metastatic potentials by spectral karyotyping, comparative genomic hybridization, and a novel method of positional expression profiling. Cancer Res 2002;62: 6981-6989.

51 Seraj MJ, Harding MA, Gildea JJ, et al. The relationship of BRMS1 and RhoGDI2 gene expression to metastatic potential in lineage related human bladder cancer cell lines. Clin Exp Metastasis 2000;18:519-525.

52 Vale RD, Reese TS, Sheetz MP. Identification of a novel force-generating protein, kinesin, involved in microtubule-based motility. Cell 1985;42:39-50.

53 Endow SA. The emerging kinesin family of microtubule motor proteins. Trends Biochem Sci 1991;16: 221-225.

54 Yamazaki H, Nakata T, Okada Y, et al. Cloning and characterization of KAP3: a novel kinesin superfamilyassociated protein of KIF3A/3B. Proc Natl Acad Sci USA 1996;93:8443-8448.

55 Kaverina IN, Minin AA, Gyoeva FK, et al. Kinesinassociated transport is involved in the regulation of cell adhesion. Cell Biol Int 1997;21:229-236.

56 Kaverina I, Krylyshkina O, Small JV. Microtubule targeting of substrate contacts promotes their relaxation and dissociation. J Cell Biol 1999;146: 1033-1043.

57 Krylyshkina O, Kaverina I, Kranewitter W, et al. Modulation of substrate adhesion dynamics via microtubule targeting requires kinesion-1. J Cell Biol 2002; 156:349-359. 